\title{
Standard and immunohistochemical staining methods for diagnosis of cervical lymph node metastasis in nasopharyngeal carcinoma
}

\author{
N.D. Phuc ${ }^{1}$, L.M. Ky ${ }^{2}$, N.T. Binh ${ }^{2}$, N.T. $\operatorname{Tuan}^{3}$, N.T.K. Van ${ }^{1}$, \\ T.X. Hai ${ }^{4}$ and V.T.M. Thuc ${ }^{5}$ \\ ${ }^{1}$ National Ear Nose and Throat Hospital, Vietnam. \\ 2 Thaibinh Pharmacy-Medical University, Vietnam \\ ${ }^{3}$ Vinh Medical University-Nghe An-Vietnam \\ ${ }^{4}$ Nghe An Maternity - Pediatric Hospital, Vietnam \\ ${ }^{5}$ Tam Anh Hospital, Vietnam \\ Corresponding author: V.T.M. Thuc \\ E-mail: vuminhthuc2010@yahoo.com.vn
}

Genet. Mol. Res. 20 (1): gmr18735

Received December 30, 2019

Accepted April 20, 2020

Final Revision January 25, 2021

Published March 29, 2021

DOI http://dx.doi.org/10.4238/gmr18735

\begin{abstract}
Nasopharyngeal carcinoma (NPC) is a rare cancer in the Western hemisphere (1 case per 100,000 individuals). Conversely there are some areas, such as Southern China, Southeast Asia (Vietnam), the Mediterranean basin and Alaska, where morbidity reaches 80 per 100,000 individuals. Detecting cervical lymph node metastasis is important because it helps recommend the radiation dose and the chemotherapy strategy. We compared the diagnostic value of hematoxylin-eosin (HE) staining and a immunohistochemistry technique to determine the pathological features of tumors, in 53 samples of cervical lymphadenopathy from 30 patients with NPC. With HE, metastasis was detected in 41 of 53 samples with NPC and cervical lymphopathy. In the remaining 12 slides, the $\mathrm{HE}$ staining did not show signs of metastasis. Staining with immunohistochemistry revealted that 10 of 12 "negative" diagnoses based on HE staining, were actually metastatic. This means that $20 \%$ of the patients would not receive correct treatment based on HE staining alone. We conclude that
\end{abstract}


immunohistochemistry is a highly sensitivity technique that can detect metastases that are not diagnosed by HE staining.

Key words: Nasopharyngeal carcinoma; Immunohistochemistry; Hematoxylineosin; Lymph node; Metastasis

\section{INTRODUCTION}

NPC is the most common cancer of the head and neck, found in 5 out of 10 common malignancies in Vietnam. Each year, 250-300 patients undergo treatment at the Ear Nose and Throat Hospital in (Sharma et al., 2010). In terms of pathology, undifferentiated nasopharyngeal carcinoma (UCNT) is found at a frequency of $86 \%$ in Vietnam, $90 \%$ in Hong Kong, but only $45 \%$ in France. This feature of the NPC in the Asian region is important.

There are many studies of nasopharyngeal carcinoma (NPC) in Vietnam and in neighboring regions. The expression of the LMP-1 (Latent membrane protein), LMP-2, and miR-155 genes is being studied (Lê Trung Thọ, 2008), and possible biomarkers of this type of cancer are being searched for (Lao and Le, 2019; Lao et al., 2019). Most of the reports are based on immunohistochemistry (IHC) methodology. For example, 40 biopsy samples from the Cho Ray Hospital, Vietnam, were used in a study about miR141. All samples were submitted to histopathological analysis, and NPC was confirmed through immunohistochemistry (Lao et al., 2018).

UCNT is very sensitive to chemotherapy (Som et al., 2000) and radiation (Edge and Edge, 2017; Lao et al., 2018; Chen et al., 2019; Moon et al., 2020). However, it often metastasizes (Jiang and Wei, 2015; Wang et al., 2017; Huang et al., 2020), and the cervical lymph node is the most common site (Zhang et al., 2010; Chan et al., 2011). It is considered a poor prognosis. Lymphadenopathy near the digastric muscle ( $\mathrm{m}$. Digastricus) may be the first sign of the disease (Hsieh et al., 2011; Loh and Petersson, 2011). Therefore, a qualitative diagnosis of the prevalence of the oncological process gives a good opportunity to improve both the quality and life expectancy of patients.

\section{MATERIAL AND METHODS}

We examined 53 samples from lymph nodes with possible metastasis taken from 30 patients. All patients from whom tissue samples were taken knew about the study and agreed to it by signing informed consent forms. They also agreed with publishing the results. The study was approved by and complies with the requirements of the responsible committee for experiments with human participation (Vietnamese unit) and was conducted in accordance with the Helsinki Declaration of 1975 (with revision in 2000).

The diagnosis "nasopharyngeal cancer" of all 30 patients was confirmed and classified. Lymph nodes were examined by ultrasound and biopsy. The samples were fixed in wax for regular staining and immunohistochemistry.

This was a prospective descriptive study. Patients were selected who had an endoscopically and histologically confirmed diagnosis with clinical manifestations in the nasopharynx. All patients underwent a fine-needle biopsy of the affected lymph nodes. 
Immunohistochemistry was performed by $\mathrm{ABC}$ method (Avidin and Biotinylated horseradish peroxidase macromolecular complex).

\section{RESULTS}

\section{Results on regular staining (HE)}

Metastasis was detected in 41 of 53 samples by HE staining, and 12 samples were diagnosed as clean (Table 1).

Table 1. Results of nasopharyngeal cancer diagnosis of 53 tumors based on hematoxylin-eosin staining.

\begin{tabular}{llll}
\hline & Metastasis & No metastasis & Total \\
\hline Number of lymph nodes & 41 & 12 & 53 \\
\hline
\end{tabular}

Staining with HE helps detect atypical cells and determine their histological condition. For example, Figure 1 shows squamous cell carcinoma metastasis.

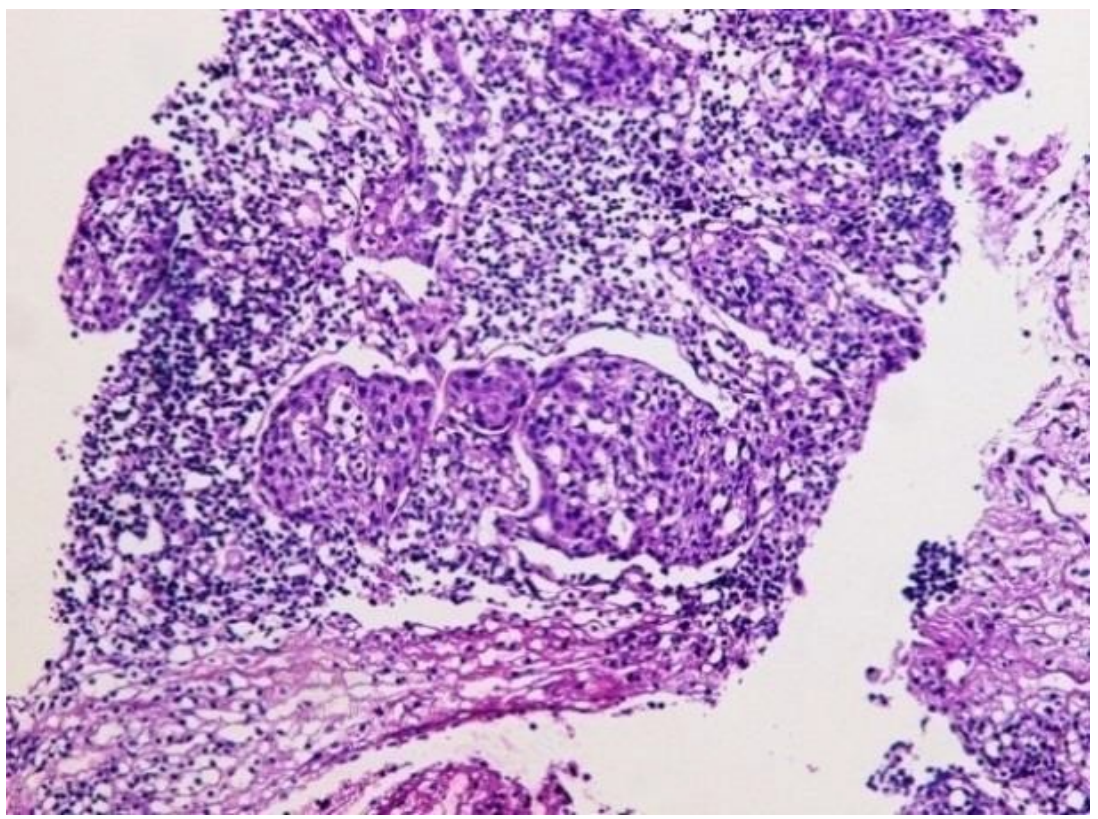

Figure 1. Squamous cell carcinoma metastasis visualized with hematoxylin-eosin staining (magnification 100X).

\section{Results of immunohistochemistry stain}

Metastasis was detected in 51 of 52 lymph nodes. All samples where metastasis was recorded based on HE staining were also positive at IHC staining. The proportion of metastasis-negative samples was only 2 in 53. 
Table 2. Results of immunohistochemistry staining of 53 nasopharyngeal tumors.

\begin{tabular}{llll}
\hline Results & Positive (n) & Negative (n) & Total \\
\hline Lymph node metastasis & 51 & 2 & 53 \\
\hline
\end{tabular}

To increase the sensitivity of the IHC method, the biomarker cytokeratin (CK) was used. With a positive reaction to cytokeratin, the cells give a brown color of different intensities. Figure 2 shows a microscopic slide where normal lymphocytes are stained with blue and squamous cell carcinoma metastasis are marked with brown.

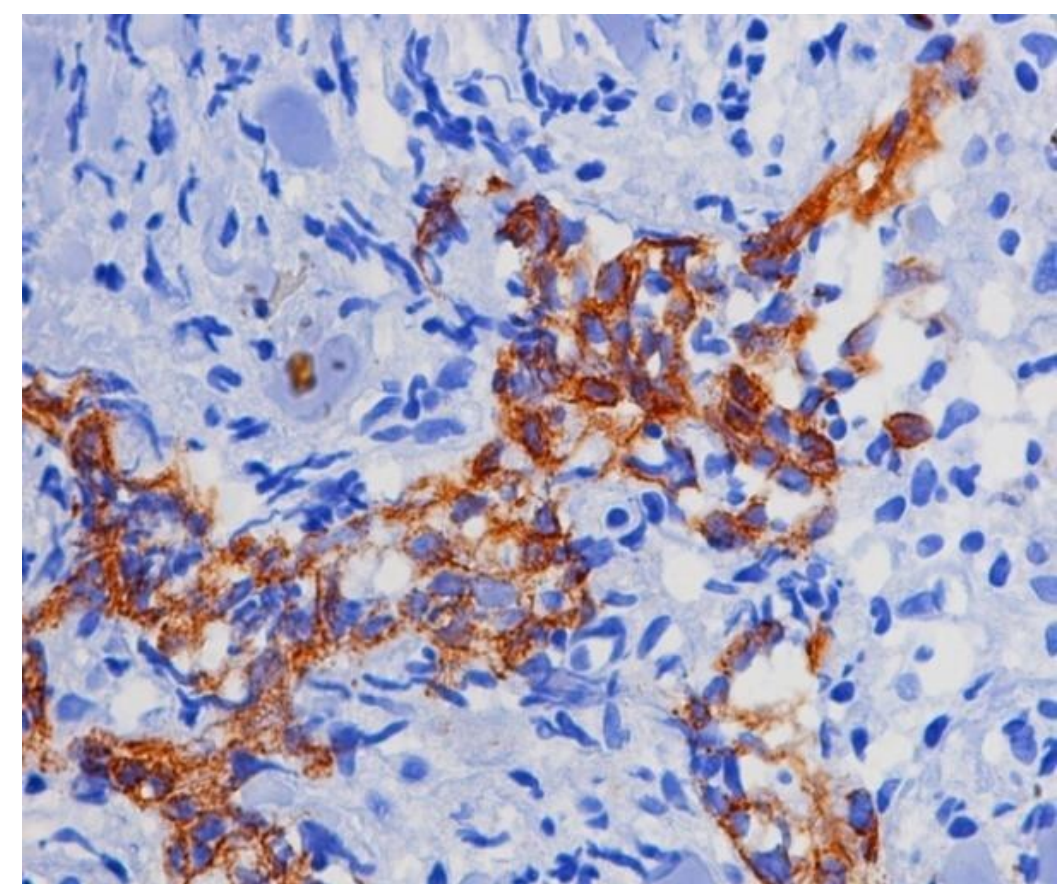

Figure 2. Normal lymphocytes stain negative with cytokeratin (blue) and positive for squamous cell metastasis with cytokeratin mark (brown). x100 magnification.

\section{Comparing the results of regular staining and immunohistochemistry}

HE staining did not reveal atypia in 10 samples of lymph nodes affected by metastasis, and $83 \%$ of "negative" slides in fact were positive. When recounting, it turns out that undiagnosed metastasis remained in 6 patients, which is $20 \%$ (Table 3 ).

Table 3. Results of immunohistochemistry compared with hematoxylin-eosin staining of 53 nasopharyngeal tumors.

\begin{tabular}{llll}
\hline Method & Positive (n) & Negative (n) & Total \\
\hline Hematoxylin-eosin stain & 41 & 12 & 53 \\
Immunohistochemistry stain & 51 & 2 & 53 \\
\hline
\end{tabular}


All of the 41 lymph nodes showing metastasis on HE staining were also positive with immunohistochemistry stain cytokeratin (CK) marking. The concordance of the two methods was $100 \%$.

The IHC technique stains atypical cells much brighter, and it is not difficult to examine them under a microscope. For comparison, in Figures $3 a$ and $3 b$, the same slides with carcinoma metastasis, stained differently.

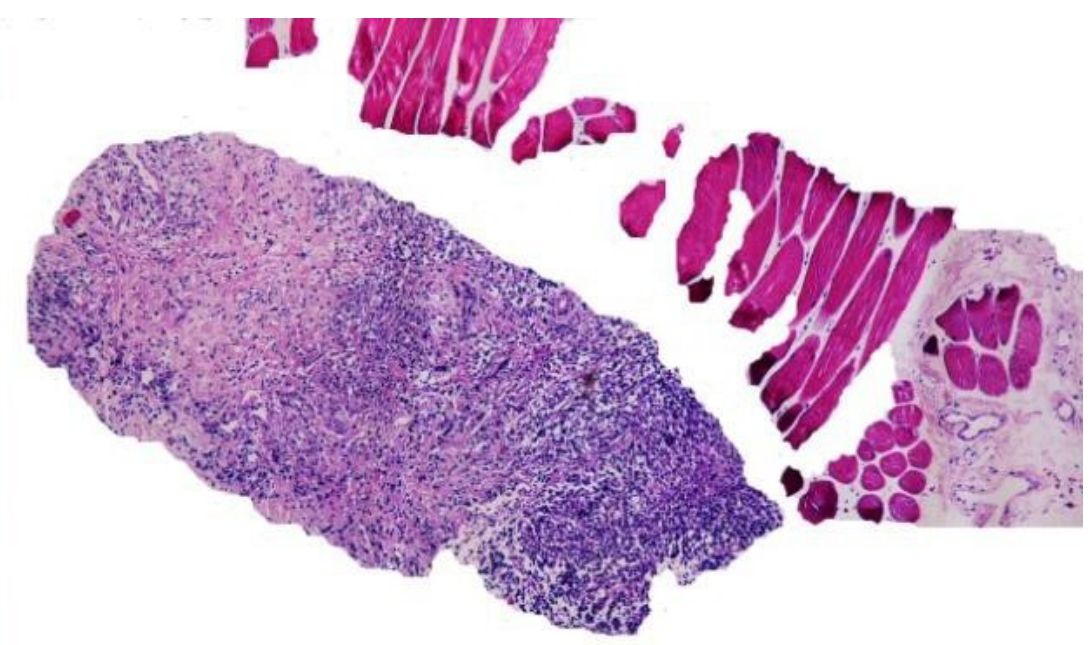

Figure 3a. Sclerosis region of a lymph node and metastasis region. Hematoxylin-eosin staining x 100 .

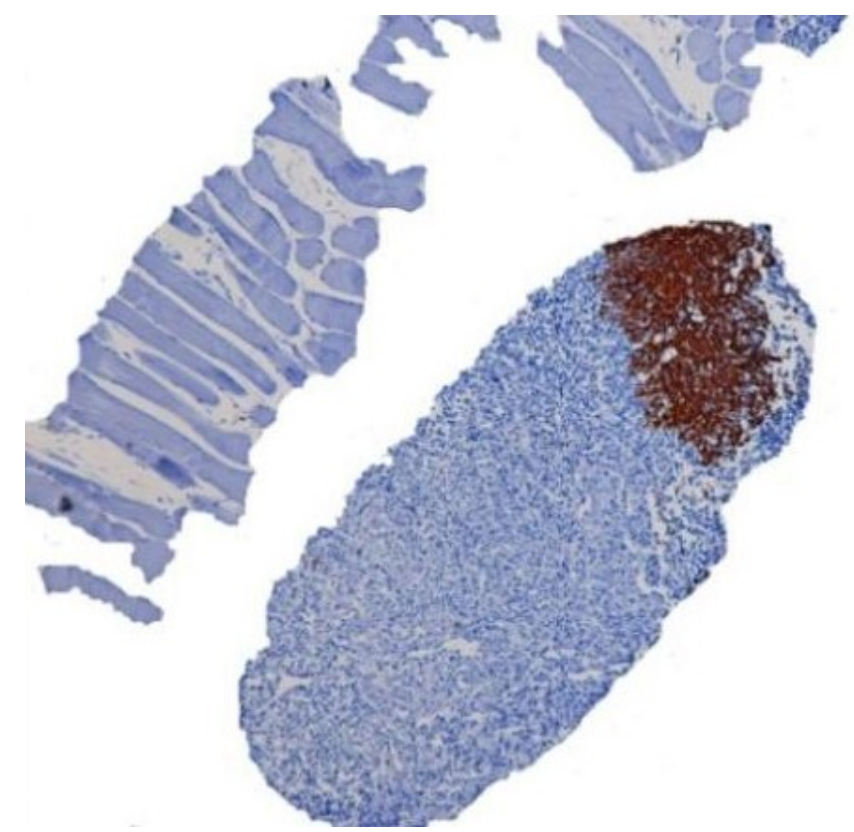

Figure 3b. Immunohistochemistry staining with cytokeratin (CK) marking x 100. Sclerosis region of a lymph node (blue) and metastasis region highly positive with CK mark (brown). 
The IHC technique allows one to consider micro metastasis, the identification of which is impossible when working with $\mathrm{HE}$ staining. In figures $4 \mathrm{a}$ and $4 \mathrm{~b}$ examples of micro metastasis.

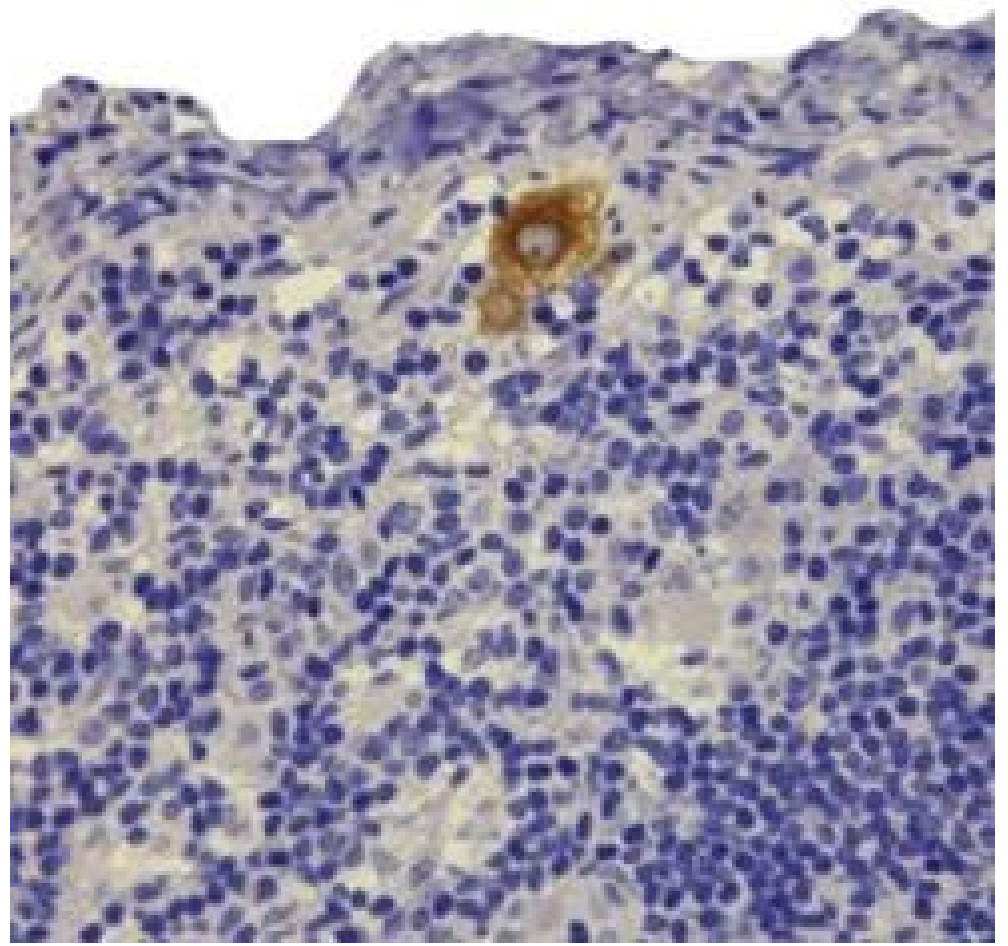

Figure 4a. Micro metastasis, only discovered on immunohistochemistry with cytokeratin marking. x 100 .

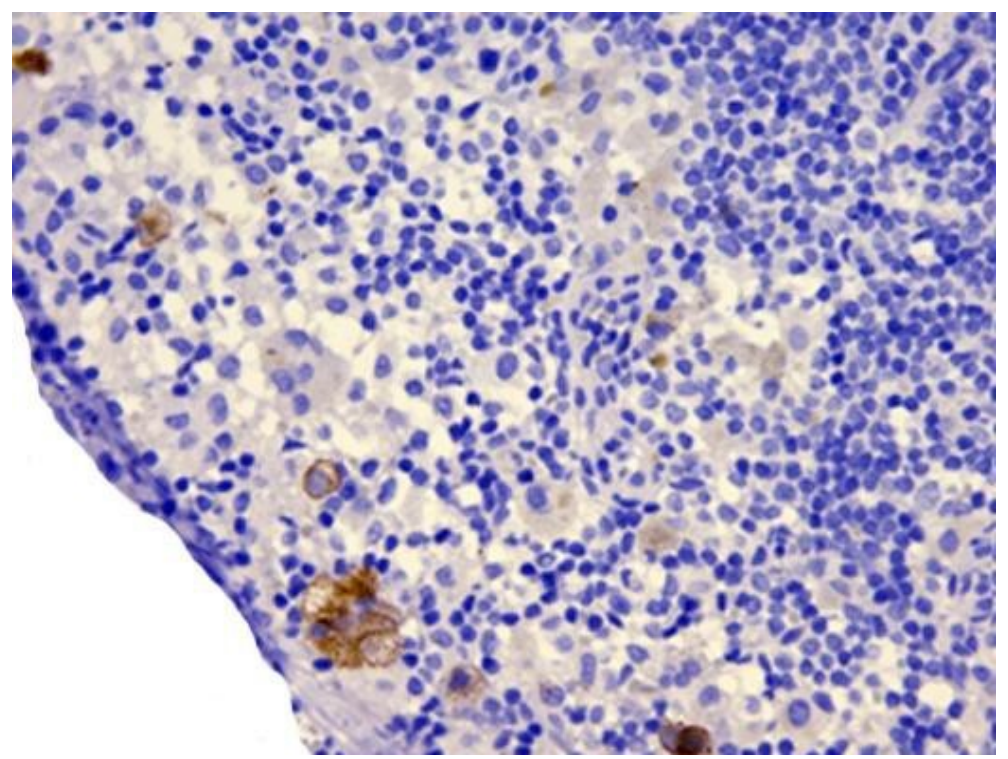

Figure 4b. Micrometastasis, only discovered on immunohistochemistry stain with cytokeratin marking x 100 . 


\section{DISCUSSION}

\section{The results of regular pathology analyses}

Nowadays, there are many methods to identify metastasis in lymph nodes (scintigraphy, CT (computed tomography) scanner, PET-CT (positron emission tomography - computed tomography), etc.). These methods are used to examine almost all lymph nodes in deep anatomical positions (mediastinum, lung's hilar, head of pancreas) that cannot be biopsied (Ferlito, 1993; Cerilli et al., 2001; Li et al., 2015). But these methods are not considered as "gold method", because they are not sufficient to prove the cancer cells presentation.

Lymph node biopsy and histological research are the utmost requirement to have correct diagnosis. In the sample (fixed in wax and stained) the pathologist may see that lymph node structures are not normal, but are replaced by cancer cells. The number of tumor cells depends on the time of metastasis, degree of invasion, and subtype of the tumor (King et al., 2000; Ferlito et al., 2002; Ma et al., 2007).

Pathologists all over the world accept that regular stain can be enough for lymph node metastasis diagnosis. Metastatic cells from nasopharyngeal carcinoma often accumulate in clusters, usually in a peripheral region (following incoming lymph vessels) with different cellular features. The cells have large basophilic cytoplasm and an atypical nucleus. In the primary focus the tumor is undifferentiated type but in lymph node the cell is differentiated to some degree, and easier to recognize.

However, there are cases when staining with HE is not enough. And predict this case advance is extremely difficult or impossible. For example, various researchers e concluded that the chance to detect microcarcinomas containing three cells, is only $3 \%$ (Shanmugaratnam, 1991; Ferlito, 1993; Cerilli et al., 2001).

\section{The results of immunohistochemistry staining}

Immunohistochemistry was first introduced almost 80 years ago. In 1941, Coon et al. published the first article about method of immunoluminescence. This technique showed that antibodies binding to luminescent marks permit detection of the antigen in the tissue (Shanmugaratnam et al., 1978). Nowadays, the effectiveness of this method has been proven by many studies and cannot be challenged. And it is confirmed in our study too.

The origin of cancer cells in NPC is from epidermoid cells, and the cells in lymph node are lymphocytes (Chen et al., 2017). We used antibodies against Keratin of the epidermoid cell to detect even small quantity of cancer cells (1-3 cells). Only the epidermoid cells are positive (antibody-antigen reaction) while the lymphocytes are completely negative (it doesn't have this antigen so there is no antibody-antigen reaction) (Ohno et al., 2013; Huang et al., 2020).

Protein filaments include keratin, vimentin, desmin, triplet neurofilament protein, glial fibrinaric acid protein (GFAP). Lamine and Nestin are in the group of threads. Although these proteins have a common epitope, mono- and polyclonal types are found, and specific filaments exist for each protein (Zhang et al., 2010).

Keratin is part of medium-sized fibers (about $10 \mathrm{~nm}$ in diameter). This is the structure that forms the framework of eukaryotic cells. Keratin differs from actin in 
size and structure; it has microtubules and myosin. Keratin has long been recognized as a sign of epidermoid cells.

The significance of keratin present in tumor cells is based on the theory of the distribution of keratin in tumor and normal cells. High molecular weight keratin is often present in multilayer squamous and keratinized epidermoid cells (in the skin). Low molecular weight keratin is distributed in the simple epithelium and glandular epithelium.

The keratin protein in the cell is often called cytokeratin, unlike keratin, which is present in the cell-free layer of the multilayer planar epidermoid. Detecting a reaction with cytokeratin helps to identify the epithelial origin of the tumor.

All of 53 lymph nodes are stained immunohistochemistry with cytokeratin marker. The results showed that 41 slides were positive with regular and cytokeratin stain. In 12 cases, which were negative on regular stain, immunohistochemistry stain found out 10 metastasis, 2 other left were hyperplasia of the lymphoid tissue. According on this data, regular stain with $\mathrm{HE}$ has missed $18.9 \%$ lymph node metastasis.

\section{CONCLUSIONS}

HE staining found 41/53 lymph node carcinoma metastasis, and 12 lymph nodes were considered clean.

The immunohistochemistry stain with a CK marker showed positive in all 41 cases. The concordance between the two methods was $100 \%$.

In 12 negative samples (by $\mathrm{HE}$ ), 10 cases were positive on immunohistochemistry staining with a CK marker. So, IHC technique detected over $80 \%$ more cases than $\mathrm{HE}$ staining.

Though the histological type and adenopathy are crucial in the treatment and prognosis about $20 \%$ patients were falsely staged, leading to false treatment strategies. This was not at mistake by pathologists, because the tumor cells were extremely small. Identification of such small cells is beyond the ability of human eye even with a microscope. From the pathologist's point of view, there are not only mature cells in lymph node but also other cells (stem cells, macrophages, histocytes). It is very difficult to identify these cells with only one or a few tumor cells.

With the above results, we realized that although IHC is more expensive, it provides a more accurate diagnosis. IHC stain should be done for adenopathy, not only in NPC but also for all other tumors.

\section{ACKNOWLEDGMENTS}

The authors would like to thank the National ENT Hospital for financial support.

\section{CONFLICTS OF INTEREST}

The authors declare no conflict of interest. 


\section{REFERENCES}

Cerilli LA, Holst VA, Brandwein MS, Stoler MH, et al. (2001). Sinonasal undifferentiated carcinoma: immunohistochemical profile and lack of EBV association. Am. J. Surg. Pathol. 25(2): 156-163. DOI:10.1097/00000478- 200102000-00003.

Chan YW, Fun Lee VH, Yu Chow VL, Howe To VS, et al (2011). Extracapsular lymph node spread in recurrent nasopharyngeal carcinoma. Laryngoscope. 121(12): 2576-2580. DOI:10.1002/lary.22380.

Chen X, Zhu X, Liang Z, Li L, et al. (2017). Long-term outcomes of neoadjuvant chemotherapy followed by concurrent chemoradiotherapy (CCRT) vs CCRT alone for nasopharyngeal carcinoma in the era of intensity-modulated radiation therapy using propensity score matching method. OncoTargets Ther. 10: 2909-2921. DOI:10.2147/OTT.S135590.

Chen YP, Chan AT, Le QT, Blanchard P, et al. (2019). Nasopharyngeal carcinoma. Lancet. 394: 64-80. DOI:10.1016/S0140- 6736(19)30956-0.

Edge SB and Edge SB (2017). AJCC Cancer Staging Manual. 8th Ed. Springer. DOI:10.2214/AJR.14.12936.

Ferlito A (1993). The World Health Organization's Revised Classification of Tumours of the Larynx, Hypopharynx, and Tracheax. Ann. Otol. Rhinol. Laryngol. 102(9): 666-669. DOI:10.1177/000348949310200903.

Ferlito A, Shaha AR and Rinaldo A (2002). Retropharyngeal lymph node metastasis from cancer of the head and neck. Acta Otolaryngol. 122: 556-560. DOI:10.2214/AJR.14.12936.

Hsieh TC, Wu YC, Hsu CN, Yang CF, et al. (2011). Lower neck neurilemmoma can masquerade as lymph node metastasis on FDG PET/CT in patient with nasopharyngeal carcinoma. Clin. Nucl. Med. 36(12): e217-219. DOI:10.1097/RLU.0b013e31822920db.

Huang T, Su N, Zhang X, Ma S, et al. (2020). Systemic chemotherapy and sequential locoregional radiotherapy in initially metastatic nasopharyngeal carcinoma: Retrospective analysis with 821 cases. Head Neck. 42: 1970-1980. DOI: 10.1002/hed.26130.

Jiang X and Wei L (2005). Nasopharyngeal carcinoma and cervical masses. Lin chuang er bi yan hou ke za zhi [J. Clin. Otorhinolar.]. 19(4): 160-162.

King AD, Ahuja AT, Leung SF, Lam WW, et al. (2000). Neck node metastases from nasopharyngeal carcinoma: MR imaging of patterns of disease. Head Neck. 22(3): 275-281. DOI: 10.1002/(sici)1097-0347(200005)22:3<275::aidhed10>3.0.co;2-n.

Lao TD and Le TAH (2019). Association Between LMP-1, LMP-2, and miR-155 Expression as Potential Biomarker in Nasopharyngeal Carcinoma Patients: A Case/Control Study in Vietnam. Gen. Test. Molec. Biomark. 23(11): 815822. DOI:10.1089/gtmb.2019.0089.

Lao TD, Nguyen TAH, Ngo KD, Thieu HH, et al. (2019). Molecular Screening of Nasopharyngeal Carcinoma: Detection of LMP-1, LMP-2 Gene Expression in Vietnamese Nasopharyngeal Swab Samples. Asian Pac. J. Cancer Prevent. 20(9): 2757-2761. DOI:10.31557/APJCP.2019.20.9.2757.

Lao TD, Nguyen TV, Nguyen DH, Nguyen MT, et al. (2018). miR-141 is up-regulated in biopsies from Vietnamese patients with nasopharyngeal carcinoma. Braz. Oral Res. 32:e126. DOI:10.1590/1807-3107bor-2018.vol32.0126.

Lê TT (2008). Hóa mô miễn dịch ứng dụng trong chẩn đoán bệnh, Tài liệu tập huấn lớp đào tạo lại. [ Applied immunohistochemistry for diagnosis, Training material for re-training course]. Ha Noi: Friendship Hospital.

Li YZ, Xie CM, Wu YP, Cui CY, et al. (2015). Nasopharyngeal carcinoma patients with retropharyngeal lymph node metastases: a minimum axial diameter of $6 \mathrm{~mm}$ is a more accurate prognostic predictor than $5 \mathrm{~mm}$. Am. J. Roentgenol. 204(1): 20-23. DOI:10.2214/AJR.14.12936.

Loh KS and Petersson F (2011). Nonexophytic nasopharyngeal carcinoma: high frequency of advanced lymph node and distant metastasis. Otolaryngol. Head Neck Surg. 145(4): 594-598. DOI: 10.1177/0194599811411141.

Ma J, Liu L, Tang L, Zong J, et al. (2007). Retropharyngeal lymph node metastasis in nasopharyngeal carcinoma: prognostic value and staging categories. Clin. Cancer Res. 13: 1445-1452. DOI:10.2214/AJR.14.12936.

Moon HM, Hippenmeyer S, Luo L and Wynshaw-Boris A (2020). LIS1 determines cleavage plane positioning by regulating actomyosin-mediated cell membrane contractility. Elife. 9: e51512. DOI:10.7554/eLife.51512.

Ohno T, Thinh DHQ, Kato S, Devi CB, et al. (2013). Radiotherapy concurrently with weekly cisplatin, followed by adjuvant chemotherapy, for N2-3 nasopharyngeal cancer: a multicenter trial of the Forum for Nuclear Cooperation in Asia. J. Radiat. Res. 54(3): 467-473. DOI:10.1093/jrr/rrs115.

Shanmugaratnam K (1991). Histological typing of tumors of the upper respiratory tract and ear. 2nd ed. Berlin: Springer-Verlag. DOI:10.1007/978-3- 642-84474-4.

Shanmugaratnam K, Sobin LH and World Health Organization (1978). Histological typing of upper respiratory tract tumours. In K. Shanmugaratnam, in collaboration with L.H. Sobin and pathologists in 10 countries. Histological typing of upper respiratory tract tumours. (p. 14). Geneve.

Sharma M, Bartlett E and Yu E (2010). Metastatic retropharyngeal lymph nodes in nasopharyngeal carcinoma: imaging criteria. Exp. Rev. Anticancer Ther. 10(11): 1703-1706. DOI:10.1586/era.10.159.

Som PM, Curtin HD and Mancuso AA (2000). Imaging-based nodal classification for evaluation of neck metastatic adenopathy. Am. J. Roentgenol. 174(3): 837-844. DOI:10.2214/AJR.14.12936. 
Wang L, Guo Y, Xu J, Chen Z, et al. (2017). Clinical analysis of recurrence patterns in patients with nasopharyngeal carcinoma treated with intensity-modulated radiotherapy. Ann. Otol. Rhinol. Laryngol. 126(12): 789-797. DOI: $10.1177 / 0003489417734229$.

Zhang GY, Liu LZ, Wei WH, Deng YM, et al. (2010). Radiologic criteria of retropharyngeal lymph node metastasis in nasopharyngeal carcinoma treated with radiation therapy. Radiology. 255(2): 605-612. DOI:10.2214/AJR.14.12936. 